\title{
Three-dimensional ultrasonographic imaging of Mikulicz's disease
}

\author{
Akitake Suzuki $^{1} \odot \cdot$ Naoko Nagata $^{2} \cdot$ Miho Ohshima $^{1} \cdot$ Takeshi Suzuki $^{1} \cdot$ Nobuyoshi Minemura $^{1} \cdot$ Masanobu Yoshida $^{1}$
}

Received: 6 July 2021 / Revised: 17 September 2021 / Accepted: 20 September 2021 / Published online: 24 September 2021

(c) International League of Associations for Rheumatology (ILAR) 2021

\section{Presentation}

A 72-year-old male presented to our hospital with a 4-month history of hoarseness and bilateral swelling in the lower jaw. A non-tender broad bean-sized swelling of the bilateral submandibular glands was noted and laboratory examinations revealed a normal CRP level and elevated immunoglobulin G4 (IgG4) level of $305 \mathrm{mg}$ per deciliter. Ultrasonography of the submandibular glands showed multiple hypoechoic areas of a nodal pattern with high vascularity (Fig. 1a). Threedimensional image reconstruction of ultrasonographic findings revealed dendritically expanded and distended vessels throughout the glands (Fig. 1b). The abundant infiltration of IgG4-positive mononuclear cells with extensive fibrosis and obliterative phlebitis of the glands was histologically detected. Perivascular lesions in the large arteries were observed on contrast-enhanced computed tomographic images. Based on these findings, the patient was diagnosed with IgG4-related disease. The patient was treated with $40 \mathrm{mg}$ of prednisolone daily and was discharged on day 24 in good condition. Two months after the initiation of treatment, the patient continued to receive $20 \mathrm{mg}$ of prednisolone, and the marked regression of vessel branches was confirmed on follow-up ultrasonography (Fig. 1c, d).

\section{Discussion}

Ultrasonography has been widely used to diagnose diseases involving the salivary glands [1,2]. Mikulicz's disease is IgG4-related dacryoadenitis and sialadenitis. Submandibular gland sonography is a useful imaging modality for its diagnosis because it detects characteristic nodal/reticular changes [3]. Doppler imaging of the salivary glands shows high vascularity, which is also a hallmark of Mikulicz's disease [3, 4].

We performed three-dimensional ultrasonography on our patient after obtaining typical sonographic images of numerous nodal changes with high vascularity. Dendritically expanded and distended vessels were observed throughout the glands and appeared to originate from a glandular branch of the facial artery (white arrow). Therefore, we speculate that

Akitake Suzuki

akitake.suzuki@gmail.com

Naoko Nagata

naoko.nagata@medical.canon

Miho Ohshima

momoshima1@gmail.com

Takeshi Suzuki

tsuzuki.mitsuihosp@gmail.com

Nobuyoshi Minemura

ici01059mbj@gmail.com

Masanobu Yoshida

msnb-ysd@hotmail.co.jp

1 Department of Rheumatology, Mitsui Memorial Hospital, 1 Kanda-izumi-cho, Chiyoda-ku, Tokyo 101-8643, Japan

2 Canon Medical Corporation, 1385 Shimoishigami, Otawara-shi, Tochigi 324-8550, Japan 
a

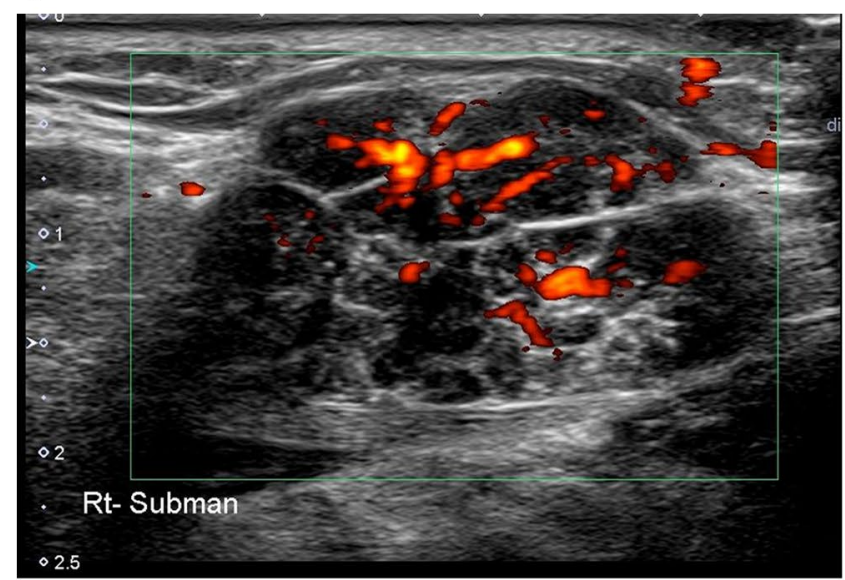

C

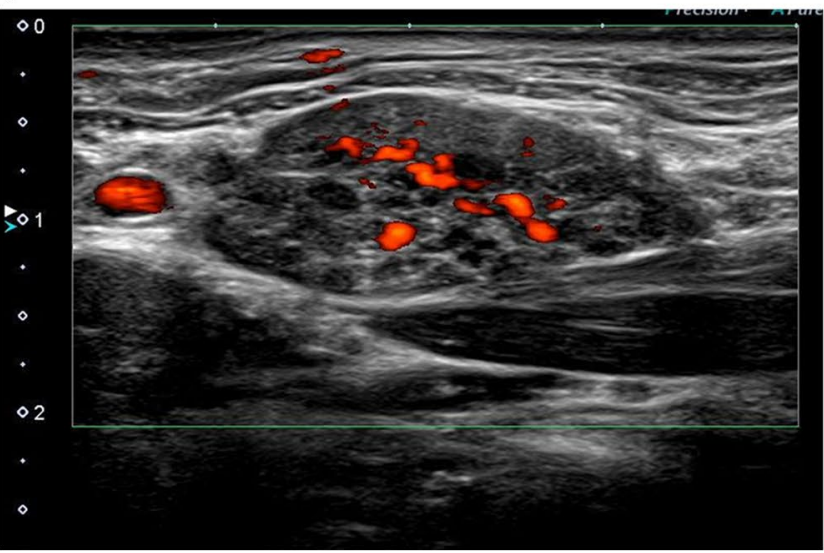

Fig. 1 a Doppler sonography of submandibular glands showing multiple hypoechoic areas of a nodal pattern with high vascularity. b Three-dimensional image reconstruction of submandibular gland sonography showing dendritically expanded, distended, and abundant vessels potentially originating from a glandular branch of the facial

the high vascularity characteristic of Mikulicz's disease originates from abundant vascular feeders from the feeding artery.

Author contribution All authors contributed equally to the conception and design of this submission. NN obtained impressive 3D pictures, MO, TS, NM, and MY drafted, read, edited, and approved the submission in its final form.

\section{Declarations}

\section{Ethics approval N/A.}

Consent for publication We obtained written informed consent from the patient for publication of this case report together with any accompanying images.

Disclosures None. b

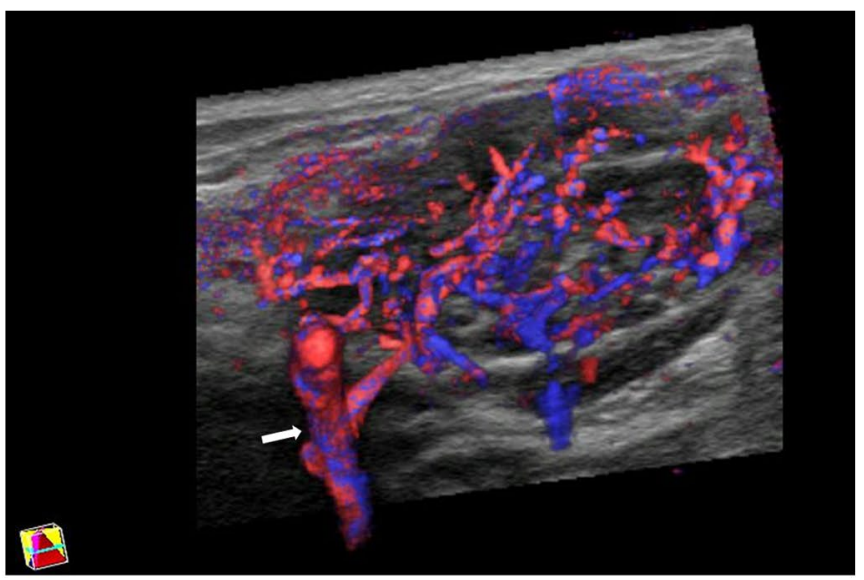

d

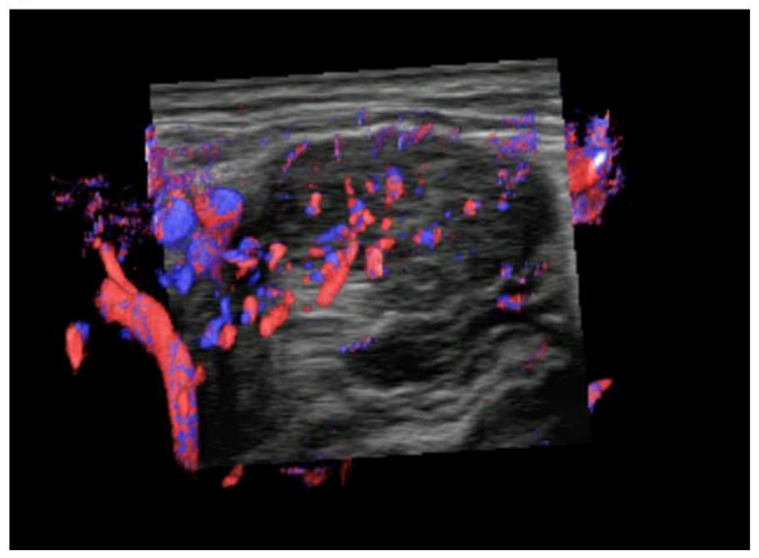

artery. The white arrow indicates the glandular branch of the facial artery. c Doppler sonography 2 months after the initiation of prednisolone showing a reduced gland size and vessel shrinkage. $\mathbf{d}$ A threedimensional image 2 months after treatment showing the regression and discontinuation of vessels

\section{References}

1. Carotti M, Ciapetti A, Jousse-Joulin S (2014) Ultrasonography of the salivary glands: the role of grey-scale and colour/power Doppler. Clin Exp Rheumatol 32:S61-70

2. Jousse-Joulin S, D’Agostino MA, Nicolas C (2019) Video clip assessment of a salivary ultrasound scoring system in Sjogren's syndrome using consensual definitions: an OMERACT ultrasound working group reliability exercise. Ann Rheum Dis 78:967-973

3. Shimizu M, Okamura K, Kise Y (2015) Effectiveness of imaging modalities for screening IgG4-related dacryoadenitis and sialadenitis (Mikulicz's disease) and for differentiating it form Sjogren syndrome (SS), with an emphasis on sonography. Arthritis Res Ther 17:223

4. Sakamoto M, Moriyama M, Shimizu M (2020) The diagnostic utility of submandibular gland sonography and labial salivary gland biopsy in IgG4-related dacryoadenitis and sialadenitis: its potential application to the diagnostic criteria. Mod Rheumatol 30:379-384

Publisher's note Springer Nature remains neutral with regard to jurisdictional claims in published maps and institutional affiliations. 